\title{
Reduction of Inter-Symbol Interference Using Artifical Neural Network System in Multicarrier OFDM System
}

\author{
Jyoti Makka ${ }^{1}, *$ Dr. Himanshu Monga, Silki Baghla \\ ${ }^{1,2,3}$ Dept. of Electronics and Communication Engineering, J.C.D.M. College of Engineering, Sirsa, Haryana, \\ India
}

Received: 31 August 2017; Accepted: 10 November 2017; Published: 08 September 2018

\begin{abstract}
The work proposes Inter-Symbol Interference (ISI) reduction scheme, ISI being a major problem in Optical systems, which produces various type of non-linear distortions. So the implementation of OFDM system using Artificial Neural Network (ANN) scheme with M-QAM modulation technique is proposed and compared with the conventional OFDM system without using ANN. This proposed scheme is implementation of Backpropagation (BP) algorithm over AWGN channels to achieve an effective ISI reduction in orthogonal frequency division multiplexing (OFDM) systems. Simulation results prove that ANN equalizer can further reduce ISI effectively and provide acceptable BER and better MSE plot compared to conventional OFDM system.
\end{abstract}

Index Terms: OFDM, Artificial Neural Network (ANN), FFT, QAM, BER, ISI, MMSE

(C) 2018 Published by MECS Publisher. Selection and/or peer review under responsibility of the Research Association of Modern Education and Computer Science

\section{Introduction}

OFDM facilitates the possible growth of wireless communication for high quality multimedia services, high speed internet access, as well as data, video and voice transmission at higher rates. This multi-carrier modulation technique is limited to $4 \mathrm{G}$ mobile communication; it will also be implemented in the $5 \mathrm{G}$ communication system [1]. It also provides a possible solution to multipath frequency selective fading effect. However, the problem of ISI occurs due to causes of multipath propagation, dispersive, noisy and fading channels present in the original signal in OFDM system. If the ISI is not mitigated properly, then decision

\footnotetext{
* Corresponding author : Himanshu Monga

E-mail address: jyotiarora02nov@gmail.com, himanshumonga@gmail.com, silky.er@gmail.com
} 
device may interpret the logic wrongly [2].

In this paper, ANN is proposed and is implemented after decoder of an OFDM system and this approach minimizes the error. Further, this proposed technique is compared to conventional ISI reduction techniques.

The paper constitutes: Section II, which deals with relevant literature; Section III proposed, OFDM system description; ISI system model; Section IV explains about proposed methodology; Section V gives simulation setup and results; Section VI highlights conclusions and future scopes and Section VII presents references.

\section{Reaserch Work}

In [3] author illustrated the performance of OFDM system by the use of low symbol rate, long symbol duration of the modulation scheme as well as use of guard bands for the reduction of ISI. In [4] author proposed V-BLAST, Decision feedback signal detection method of cancellation of ISI and cyclic reconstruction method for the ICI removal purpose. In [6] author proposed zero insertion (ZI) which insert zeros and each OFDM frames contain the same length. It also compares different guard interval (cyclic prefix, zero padding and known symbol padding) insertion techniques and shows that the ZI approach reduces the transmission rate, distortion in the channel as well as a $20 \%$ data reduction in the redundant data as compared to CP. In [7] proposed zero-forcing equalizer which used longer filter span to compensate for the multipath channel distortion. In [9] highlighted CP and ZP effect on the BER and give better performance under Rayleigh fading channel as compared to AWGN. In [10] presented a two-dimensional 9/12 modulation code to combat 2D ISI and achieve $\sim 2 \mathrm{Db}$ gain as compared to without encoding modulation code. In [11] proposed unique word (UW) sequence which improved bandwidth efficiency, 15\% throughput, and spectral efficiency $17 \%$ as compared to CP but CP-OFDM give better BER than UW-OFDM system. In [12] proposed two channel estimator in which MMSE estimator gives more complexity than the LS estimator but MMSE need low SNR and LS need high for better performance.

\section{Basic OFDM System Model}

Any OFDM system consists of consider $\mathrm{N}$ subcarriers, suppose that the complex symbol $X(n)$ is the signal point from the modulation signal constellation, which is modulated on the nth subcarriers and the $x(t)$ is the transmitted signal which is defined in [5] as follows:

$$
x(t)=\sum_{n=0}^{N-1} X(n) \mathrm{e}^{\left(\mathrm{j} 2 \pi \mathrm{f}_{\mathrm{n}} \mathrm{t}\right)}, \quad 0 \leq t \leq T,
$$

Where $f_{n}=f_{0}+n / T, T=N T_{s}$, is the interval of OFDM symbol, $T_{s}$ Is the symbol data period. To synthesize the above OFDM signal in equation (1) use Inverse Fast Fourier transforms (IFFT) which samples the $x(n)$ signal with sample rate $\frac{T}{N}$. After IFFT the original time domain symbolic signal $x_{n}$ is given as below:

$$
\begin{aligned}
& x(n)=\operatorname{IFFT}\{X(K)\} \\
& \mathrm{x}(\mathrm{n})=\mathrm{x}(\mathrm{kT} / \mathrm{N})=\sum_{\mathrm{n}=0}^{\mathrm{N}-1} \mathrm{X}(\mathrm{K}) \mathrm{e}^{\mathrm{j}(2 \pi \mathrm{nk} / \mathrm{N})}, \quad \mathrm{n}=0, \ldots \ldots, \mathrm{N}-1
\end{aligned}
$$

Where $N$ is the FFT length and $X(K)$ Is the baseband data sequence. To reduce, the ISI [6] inserted cyclic prefix which extends the duration of symbols and is given as:

$$
x_{t}(n)=\left\{\begin{array}{l}
x(N+n), n=-N_{g}, N_{g}+1, \ldots \ldots,-1 \\
x(n), n=0,1,2, \ldots \ldots \ldots \ldots \ldots . \ldots .1
\end{array}\right.
$$


Then fading channel with white Gaussian noise transmitted $x_{t}(n)$ Signal and calculated the received signal $y_{t}(n)$ in the form below:

$$
y_{t}(n)=x_{t}(n) y(n)+w(n)
$$

Where, $h(n)$ is the response of impulse for selective fading channel and $w(n)$ is the AWGN. After that signal $y(n)$ will be transmitted without using $\mathrm{CP}$ is given as:

$$
\begin{aligned}
& Y(K)=F F T\{y(n)\} \\
& \mathrm{Y}(\mathrm{K})=\frac{1}{\mathrm{~N}} \sum_{\mathrm{K}=0}^{\mathrm{N}-1} \mathrm{Y}(\mathrm{n}) \mathrm{e}^{\left(-\mathrm{j} \frac{2 \pi \mathrm{nl}}{\mathrm{N}}\right)}, \quad \mathrm{n}=0, \ldots \ldots \ldots \mathrm{N}-1
\end{aligned}
$$

\section{Inter-Symbol Interference (Isi)}

In band limited region, when the number of pulses is transmitted in succession then these pulses will interfere with each other and hence receiver will not be interpret the transmitted logic. So the BER in the receiver will increase. We can visualize inter-symbol interference (ISI) effect in Fig 1.

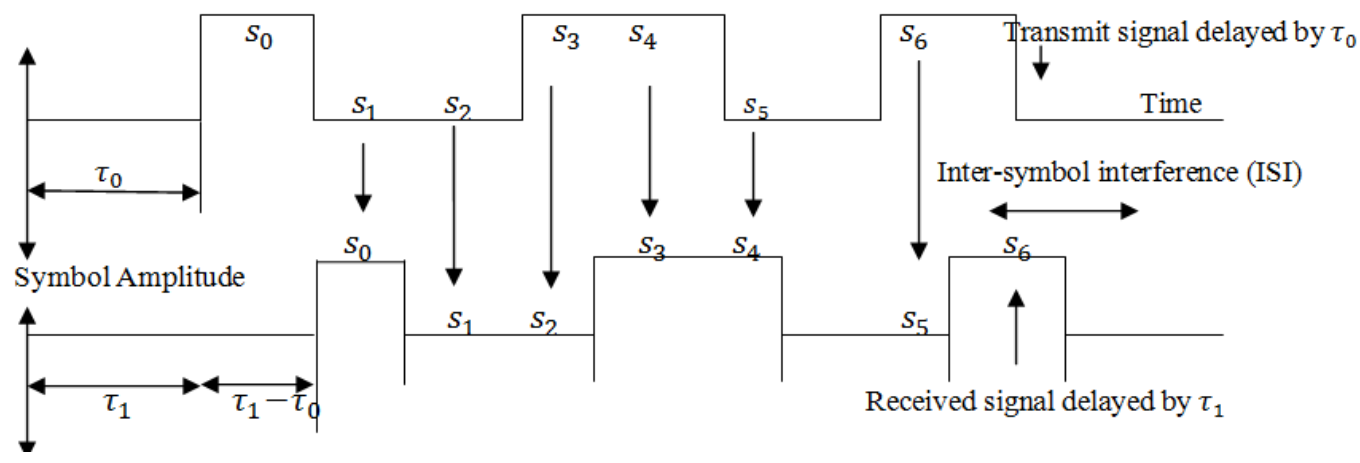

Fig.1. Inter-Symbol Interference (ISI) effect

When $\tau_{1}-\tau_{0}>T$, then different symbol creates interference with each other as shown in above figure where $\mathrm{T}$ is the symbol time, $\tau_{0}$ Is the transmitted signal delay and $\tau_{1}$ Is the received signal delay. $\tau_{1}-\tau_{0}=\sigma_{\tau}$. If $\sigma_{\tau}>T$ then this lead to inter-symbol interference (ISI) where $\sigma_{\tau}$ is the delay spread. ISI is undesirable since it leads to distortion of the original transmitted signal but if we can make $T>\sigma_{\tau}$ this implies no ISI.

The ISI channel has been described as:

$$
y(k)=h(0) x(k)+h(1) x(k-1)+v(k)
$$

Where $y(k)$ received symbol at time $\mathrm{k}, x(k)$ transmitted symbol at time $\mathrm{k}, x(k-1)$ is the previous transmitted symbol at $\mathrm{k}-1, v(k)$ is the noise signal. The past symbol $x(k-1)$ Interference with the present symbol $x(k)$ and introduce ISI.

\section{Proposed Methodology}

In this work, we have proposed an artificial neural network combined technique, which provides ISI reduction in OFDM systems. 


\subsection{Proposed OFDM with ANN}

The proposed OFDM with ANN scheme is given in below Fig. 3. Where 9600 information bits are randomly generated by using 96 bits single frame size and 100 total number of frames. Again convolution channel coder encodes the information bit by using the inputloutput size of the encoder, constraint length and specified poly2trellis $(171,133]$ matrix and provide $1 \times 192$ coded data. Matrix interleaving, block is employed to further restrict the burst error developed during transmission. The output of the interleaver is $4 \times 48$ coded output data is obtained. Further a data converter is available which convert the binary interleaving coded data into decimal format.

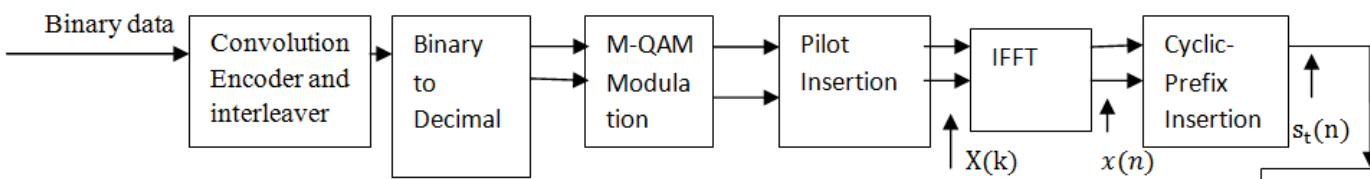

AWGN Channel

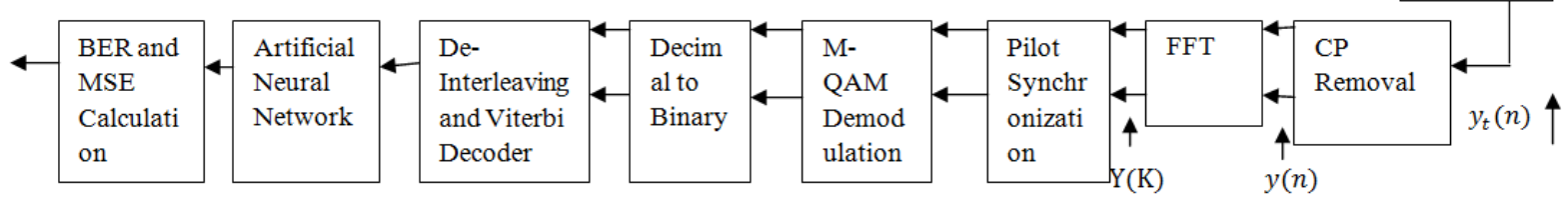

Fig.2. Block diagram of proposed (OFDM-ANN) technique

After that, M-QAM modulation scheme, the symbol values are generated. The proposed method provides better BER performance by $(16,64$, and 256) QAM modulation schemes. This modulation value determines the number of bits $(4,6$, and 8$)$ per sub-carriers. Further, the pilot insertion concept introduced which adds data subcarrier values, 4 pilot symbols, and one DC subcarrier. Again, we take 64 points IFFT lengths which is equal to a number of carriers. The IFFT length, data subcarriers, pilot value and DC subcarrier also determine the guard band values. The 16 cyclic prefix lengths are inserted before the AWNG channel. CP means prefixing of symbols from the last part to front part.

Here, the AWGN channel transmits original information data from transmitter to receiver. It adds some noise which destroy the signal. This can only be rectified by the use of ANN technique at the receiver side as shown in Fig. 2.

\subsection{Proposed Artificial Neural Network (ANN) Algorithm}

The artificial neural network is implemented after Viterbi decoder block of an OFDM system that means it takes input from an OFDM system and output set as a target value. ANN is an artificial intelligence method used to enhance the capacity of computer by sending human like intelligence. This proposed work basically depends on back-propagation (BP) algorithm which sends signals in forward direction and propagate error in backward direction. The multilayer BP network is shown in Fig 3. And the Log-Sigmoid function in [8] is given as below:

$$
f(x)=\frac{1}{1+e^{-x}}
$$




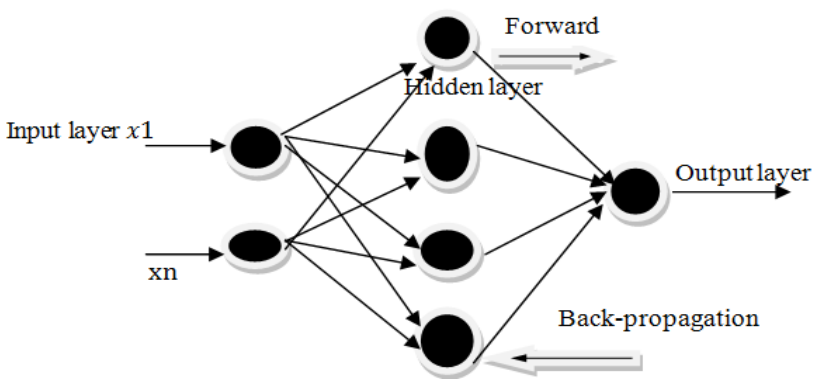

Fig.3. Multilayer back-propagation network

Fig. 4 shows, the flow chart of ANN which give Error, $e(n)$ as the difference between the target (actual) value and expected value in [10] [14] as below:

$$
e(n)=d(n)-y(n)
$$

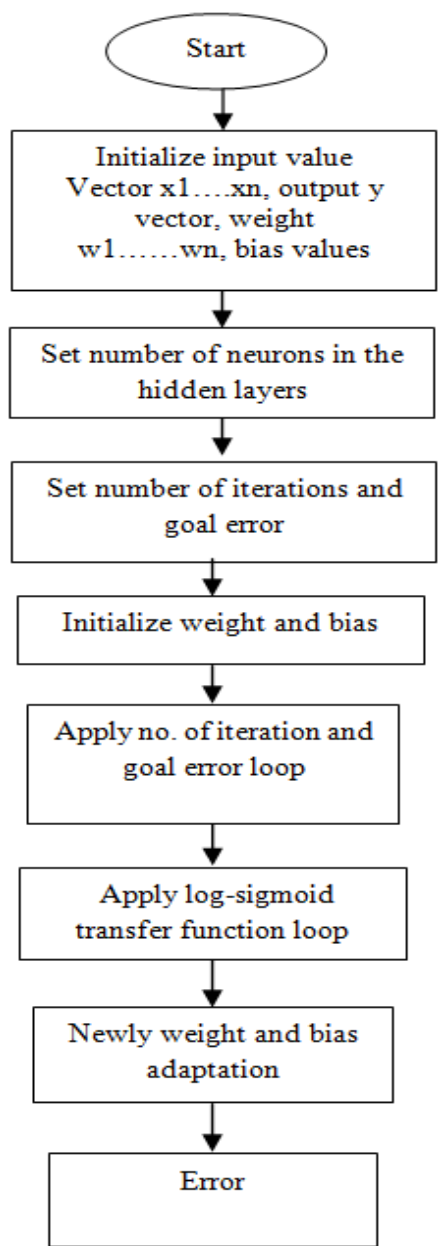

Fig.4. Flow chart of ANN algorithm 


\section{Simulation Setup and Results}

\subsection{Simulation Setup}

In this section, the simulation setup of an OFDM system is shown in Table 1and Table 2 shows the simulation setup of an ANN which evaluate the BER, MSE performances of an OFDM system.

Table 1. Parameters of OFDM system

\begin{tabular}{|c|c|}
\hline Parameter & Value \\
\hline FFT size & 64 \\
\hline Number of carriers & 64 \\
\hline Single frame size & $96 \mathrm{bits}$ \\
\hline Total number of frames & 100 \\
\hline Cyclic-prefix & 16 \\
\hline Modulation & $16,64,256 \mathrm{QAM}$ \\
\hline
\end{tabular}

Table 2. Parameters of artificial neural networks

\begin{tabular}{|c|c|}
\hline Parameter & Value \\
\hline Number of inputs & 2 \\
\hline Number of hidden layers & 1 \\
\hline Number of neurons & 90,1 \\
\hline Iteration & 1000 \\
\hline Goal error & $10 \mathrm{e}-7$ \\
\hline Training function & Back-Propagation \\
\hline Transfer function & Log- Sigmoid \\
\hline
\end{tabular}

\subsection{Simulation Results}

A. BER performances of OFDM systems with varying QAM Modulation schemes:

In Fig. 5, show the BER of conventional OFDM which gives better BER reduction performance for 16QAM modulation compared to 64 and 256 QAM modulation schemes.

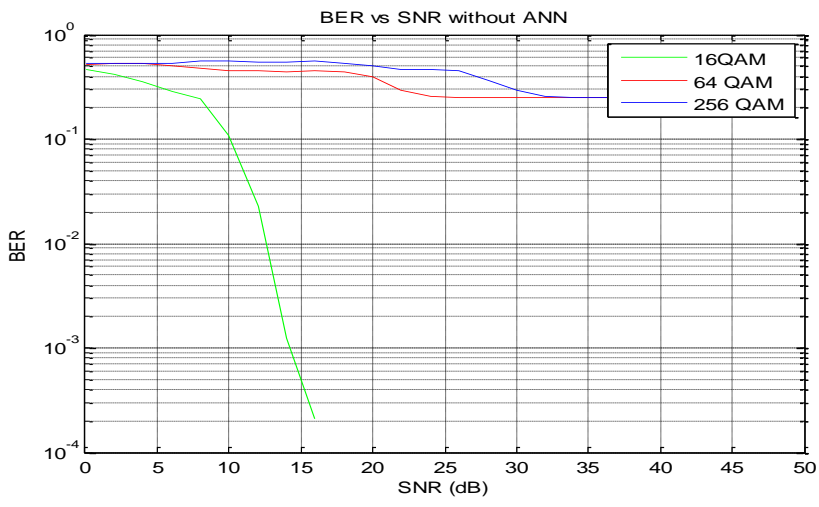

Fig.5. BER vs. SNR Graph without ANN 
B. BER performances of Proposed OFDM Systems with varying M-QAM Modulation schemes:

In Fig. 6, show the BER of proposed OFDM, which give better BER reduction performance for 256 QAM as compare to 16,64 QAM modulation schemes.

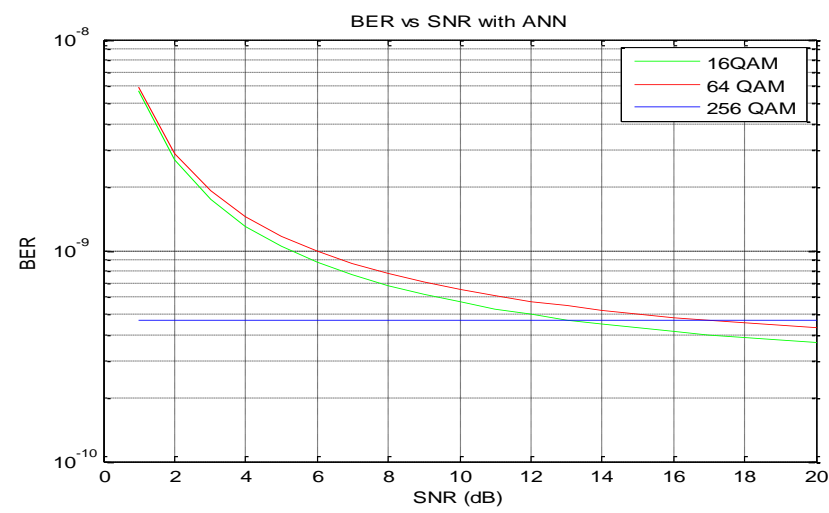

Fig.6. BER vs. SNR Graph with ANN

Table 3. BER for Conventional and Proposed OFDM systems

\begin{tabular}{|c|c|c|}
\hline Variation in M-QAM Modulation & BER for Conventional OFDM & BER for Proposed OFDM \\
\hline 16QAM & 0.0002 & 1E-9 \\
\hline 64QAM & 0.25 & $1 \mathrm{E}-9$ \\
\hline 256QAM & 0.25 & $1 \mathrm{E}-9$ \\
\hline
\end{tabular}

Table-3 shows the BER performance by varying signal to noise ratio. It shows that the OFDM-with-ANN provide BER approximate $10^{\wedge}-9$ and conventional OFDM require BER $10^{\wedge}-4$.

C. MSE performances of the Proposed OFDM Systems with varying QAM Modulation schemes

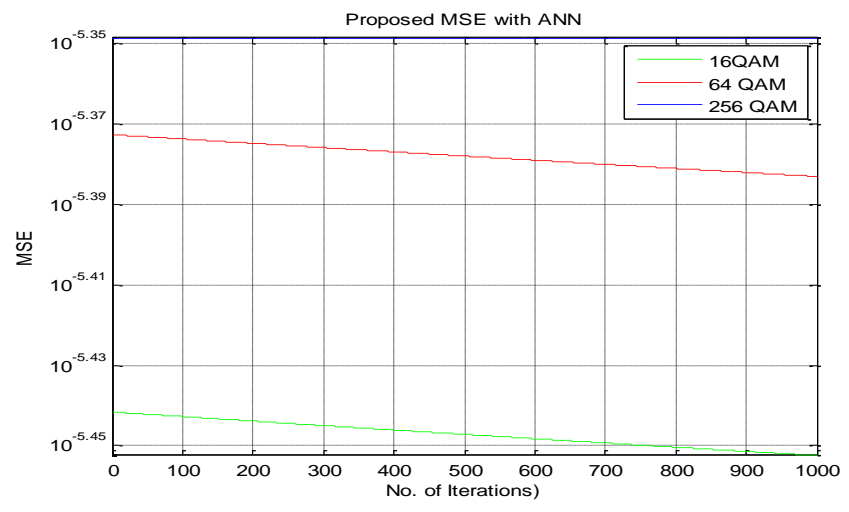

Fig.7. MSE vs. No. of iterations Graph with ANN 
Table 4. MSE for Conventional and Proposed OFDM systems

\begin{tabular}{|c|c|c|}
\hline MSE for M-QAM Modulation & Conventional OFDM & Proposed OFDM \\
\hline 16QAM & 0.05067 & 0.00000354 \\
\hline 64QAM & 0.00301 & 0.00000412 \\
\hline 256QAM & 0.49765 & 0.00000400 \\
\hline
\end{tabular}

Table-4 shows, MSE performance by varying the number of iterations. It shows that the Proposed OFDM by using M-QAM modulation require minimum MSE as compare to conventional OFDM without ANN.

\section{Conclusion and Future Scope}

This paper proposed, Back-propagation (BP) based ANN channel estimator which is further combined with OFDM system. This proposed combined technique provides better ISI reduction performance than an OFDM system without adding ANN. In this work, ISI is directly proportional to BER and inversely proportional to SNR. The future scope of this paper is to apply ANN with MIMO-OFDM system or use of other modulation technique.

\section{References}

[1] R.van Nee, R. Prasad; "OFDM for Wireless Multimedia Communications" Artech House Publishers; Boston, 2000.

[2] Z. Wang and G. B. Giannakis, Wireless multicarrier communications, IEEE Signal Processing Mag., pp. 29-48, May 2000.

[3] D.M. Bappy, Ajoy Kumar Dey, Susmita Saha; "OFDM System Analysis for reduction of Inter symbol Interference Using the AWGN Channel Platform"; IJACS; Vol. 1, No. 5, November 2010.

[4] Yang-Yang Chen, Zi-Wei Zheng; "Research on the Inter Symbol Interference Mitigation for the MIMOOFDM Systems"; IEEE 2011.

[5] Aida zaier1 and Ridha bouallègue; "a full performance analysis of channel estimation methods for time varying ofdm systems" ijmnct; Vol. 1, no. 2, December 2011.

[6] Usama S. Mohammed, Ahmed Tohamy; "A Low Complexity OFDM System with Minimum Inter-symbol Interference"; IEEE 2012.

[7] Pragya Sharma;"Performance Analysis of Zero-Forcing Equalizer for ISI Reduction in Wireless Channels"; International Journal of Engineering Research \& Technology; Vol. 1 Issue 8, October - 2012.

[8] Amit goyal1, gurleen kaur walia2 and simranjeet kaur; "implementation of back propagation algorithm using MATLAB"; international journal of information technology and knowledge management; July-Dec 2012, volume 5, no. 2, pp. 429-431.

[9] Prafulla. D. Gawande and Sirddharth. A. Ladhake; "BER Performance of OFDM system with cyclic prefix \& zero padding”; International Journal of Advances in Engineering \& Technology; Mar. 2013.

[10] Nirmalkumar S. Reshamwala1, Pooja S. Suratia, Satish K. Shah; "Artificial Neural Network trained by Genetic Algorithm for Smart MIMO Channel Estimation for Downlink LTE-Advance System"; I.J. Computer Network and Information Security, 2014, 3, 10-19; DOI: 10.5815/ijcnis.

[11] Chi Dinh Nguyen, Jaejin Lee; "Elimination of two-dimensional inter-symbol interference through the use of a 9/12 two-dimensional modulation code"; IET Communication., 2016, Vol. 10, Issue 14, pp. 1730-735.

[12] Amevi Acakpovi, Abdul-Shakud Iddrisu, Nana Yaw Asabere and John Kwofie; "Performance Comparison of Cyclic Prefix OFDM and Unique Word OFDM in the LTE Downlink"; International Journal of Computer Science and Telecommunications; Volume 7, Issue 2, February 2016. 
[13] R Srinivasa Aditya Varma, Marthy Siva Sai Krishna; “ Estimation of channel in an OFDM wireless channel using LS and MMSE techniques"; IJECET; Volume 7, Issue 3, May-June 2016, pp. 68-74.

[14] Arne Bråten; "Data editing with Artificial Neural Networks"; December 1997.

\section{Authors' Profiles}

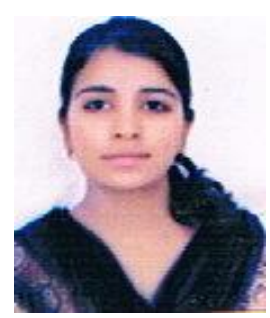

Jyoti Makkar received by B. Tech. In Electronics and Communication engineering from Eternal University, Baru Sahib (H.P) in 2014.Currently pursuing M. Tech. From J.C.D.M.College of Engineering, Sirsa, Haryana; India. Her research area is wireless communication and OFDM technology

Dr. Himanshu Monga-Obtained $\mathrm{PhD}$.from Thapar Institute of Engineering and Technology, (TU) Department Of ECE, Patiala, Punjab, INDIA, in the field of Optical networks / coding and Wireless networks. He obtained his bachelor's degree in Electronics \& Communication Engineering from Govt. Engineering College, Amravati University, Maharashtra, India and Master's degree in Electronics and Telecommunication Engineering \& Masters degree in management in Human resource management. Presently he is presently working as Director/Principal in Jan Nayak Chaudhary Devi Lal Lal Vidyapeeth, Sirsa. Before this he served as Professor and Dean Research in Lovely Professional University, Phagwara, India. He possesses work experience of 19 plus years in academics and industry/research.

He has more than 95 Research papers in international/national conferences. Have more than 22 Thomson Reuter SCI publications to his credit. He is the life member of IEEE, ISTE and reviewer of many renowned journals. He has authored 04 Books/ chapters in renowned books. He successfully completed 06 projects worth Rs 70 Lacks and successfully completed many consultancy projects.

How to cite this paper: Jyoti Makka, Himanshu Monga, Silki Baghla, " Reduction of Inter-Symbol Interference Using Artifical Neural Network System in Multicarrier OFDM System ", International Journal of Wireless and Microwave Technologies(IJWMT), Vol.8, No.5, pp. 10-18, 2018.DOI: 10.5815/ijwmt.2018.05.02 